\title{
New Contractive Conditions for Maps in G-metric Type Spaces
}

\author{
Yaé Ulrich Gaba \\ Department of Mathematics and Applied Mathematics, University of Cape Town, Rondebosch 7701, South Africa \\ Email: gabayae2@gmail.com
}

\begin{abstract}
In this article, we introduce a new type of contractive self maps defined in $G$-metric type spaces and prove a common fixed point theorem. We establish that under the convergence of a certain series, a family of self maps have a common fixed point.
\end{abstract}

Keywords: $G$-metric type space; fixed point

\section{Introduction and Preliminaries}

Fixed Point Theory is one of the most powerful and applicable areas of modern mathematics. Its applications range across numerous fields among which we can cite Optimization Theory, Convex Analysis, Convex Optimization, Approximation Theory and many more. This is due to the fact that many stability properties of most equilibrium problems can be formulated in terms of fixed points. Moreover, the underlining sets of such problems are not always the classical ones and generally the new variety of sets we encounter have not yet been investigated in detail. In recent years we have seen a considerable amount of publications in the fixed point theory for maps defined on $G$-metric spaces. The works by Mustafa and Sims $[1,2]$ were an interesting introduction to the topic. One also needs to mention the results due to Dhage [3] when defining $D$-metric spaces as a generalization of metric spaces. In our previous work [4], we introduced $G$-metric type spaces as a slight generalization of $G$-metric spaces and proved some fixed point results via what we called $\lambda$-sequences. It is the aim of this paper to give more fixed point results in the setting of $G$-metric type spaces via a new kind of contractive condition closed to that of $\lambda$-sequences. The method builds on the convergence of an appropriate series of coefficients. Recent and similar work can also be read in $[1,5,6,7,8]$.

Definition 1.1. (Compare [6]) Let $X$ be a nonempty set, and let the function $D: X \times X \rightarrow[0, \infty)$ satisfy the following properties:

(D1) $D(x, x)=0$ for any $x \in X$;

(D2) $D(x, y)=D(y, x)$ for any $x, y \in X$;

(D3) $D(x, y) \leq \alpha\left[D\left(x, z_{1}\right)+D\left(z_{1}, z_{2}\right)+\cdots+D\left(z_{n}, y\right)\right]$ for any points $x, y, z_{i} \in X, i=1,2, \ldots, n$ where $n \geq 1$ is a fixed natural number and some constant $\alpha \geq 1$.

The triplet $(X, D, \alpha)$ is called a metric type space.

Definition 1.2. (Compare [2]) Let $X$ be a nonempty set, and let the function $D: X \times X \times X \rightarrow[0, \infty)$ satisfy the following properties:

G1) $D(x, y, z)=0$ if $x=y=z$ whenever $x, y, z \in X$;

G2) $D(x, x, y)>0$ whenever $x, y \in X$ with $x \neq y$;

G3) $D(x, x, y) \leq D(x, y, z)$ whenever $x, y, z \in X$ with $z \neq y$;

G4) $D(x, y, z)=D(x, z, y)=D(y, z, x)=\ldots$, (symmetry in all three variables);

G5) $D(x, y, z) \leq K[D(x, a, a)+D(a, y, z)]$ whenever $x, y, z, a \in X$ and some constant $K \geq 1$.

The triplet $(X, D, K)$ is called a $G$-metric type space.

Moreover, for $K=1$, we recover the classical $G$-metric (see[2]). Furthermore, if $(X, D, K)$ is a $G$-metric type space, then for any $L \geq K,(X, D, L)$ is also a $G$-metric type space.

Definition 1.3. (Compare [4,2]) Let $(X, D, K)$ be a $G$-metric type space. Then a sequence $\left(x_{n}\right) \subseteq X$ is 
i) said to be G-Cauchy if for each $\varepsilon>0$ there exists $N \in \mathbb{N}$ such that $D\left(x_{n}, x_{m}, x_{m}\right)<\varepsilon$ for all $m, n \geq N$

ii) said to be $G$-convergent to $x \in X$ if $\lim _{n, m \rightarrow \infty} D\left(x, x_{n}, x_{m}\right)=0$, that is, for each $\varepsilon>0$ there exists $N \in \mathbb{N}$ such that $D\left(x, x_{n}, x_{m}\right)<\varepsilon$ for all $m, n \geq N$.

Definition 1.4. (Compare [4,2]) A G-metric type space $(X, D, K)$ is $G$-complete if every $G$-Cauchy sequence in $(X, D, K)$ is $G$-convergent.

Remark 1.5. We know (see [2]) that given a metric type space $(X, d, K)$, we can build a $G$-metric type space $(X, D, K)$ by setting

$$
D(x, y, z)=\max \{d(x, y), d(y, z), d(x, z)\}
$$

whenever $x, y, z \in X$.

Definition 1.6. ([2]) Let $X$ be a nonempty set, and let the function $D: X \times X \times X \rightarrow[0, \infty)$ be a $G$-metric on $X$. For any $x, y, z, a \in X$, we have

i) If $D(x, y, z)=0$ then $x=y=z$,

ii) $D(x, y, z) \leq D(x, x, y)+D(x, x, z)$,

iii) $D(x, y, y) \leq 2 D(y, x, x)$,

iv) $D(x, y, z) \leq D(x, a, z)+D(a, y, z)$,

v) $D(x, y, z) \leq \frac{2}{3}[D(x, y, a)+D(x, a, z)+D(a, y, z)]$

vi) $D(x, y, z) \leq D(x, a, a)+D(y, a, a)+D(z, a, a)$.

Definition 1.7. ([2]) Let $X$ be a nonempty set, and let the function $D: X \times X \times X \rightarrow[0, \infty)$ be a $G$-metric on $X$. The following are equivalent:

i) The sequence $\left(x_{n}\right)$ is $G$-convergent to $x^{*}$.

ii) $G\left(x_{n}, x_{n}, x^{*}\right) \rightarrow 0$ as $n \rightarrow \infty$.

iii) $G\left(x_{n}, x^{*}, x^{*}\right) \rightarrow 0$ as $n \rightarrow \infty$.

iv) $G\left(x_{m}, x_{n}, x^{*}\right) \rightarrow 0$ as $m, n \rightarrow \infty$.

\section{Main Results}

Let $\Phi$ be the class of continuous, non-decreasing, sub-additive and homogeneous functions $F:[0, \infty) \rightarrow$ $[0, \infty)$ such that $F^{-1}(0)=\{0\}$.

We start with the following lemma, which is a generalization of property G5 from Definition 1.2.

Lemma 2.1. Let $(X, D, K)$ be a $G$-metric type space. Then

$$
D(x, y, z) \leq K^{n}\left[D\left(x, z_{1}, z_{1}\right)+D\left(z_{1}, z_{2}, z_{2}\right)+\cdots+D\left(z_{n}, y, z\right)\right]
$$

for any points $x, y, z, z_{i} \in X, i=1,2, \ldots, n$ where $n \geq 1$.

Proof. The proof is trivial and shall be omitted.

Theorem 2.2. Let $(X, D, K)$ be a $G$-complete $G$-metric type space and $\left\{T_{n}\right\}$ be a sequence of self mappings on $X$ such that

$$
\begin{aligned}
F\left(D\left(T_{i}(x), T_{j}(y), T_{j}(z)\right)\right) \leq & F\left(\delta_{i, j}\left[D\left(x, T_{i}(x), T_{i}(x)\right)+D\left(y, T_{j}(y), T_{j}(z)\right)\right]\right) \\
& +F\left(\gamma_{i, j} D(x, y, z)\right)
\end{aligned}
$$

for $x, y, z \in X$ with $x \neq y, 0 \leq \delta_{i, j}, \gamma_{i, j}<1, i, j=1,2, \cdots$, and for some $F \in \Phi$ homogeneous with degree $s$. If

i) for each $j, \limsup _{i \rightarrow \infty} \delta_{i, j}^{s}<1$, 
ii)

$$
\sum_{n=1}^{\infty} C_{n}<\infty \text { where } C_{n}=\prod_{i=1}^{n} \frac{\delta_{i, i+1}^{s}+\gamma_{i, i+1}^{s}}{1-\delta_{i, i+1}^{s}}
$$

then $\left\{T_{n}\right\}$ has a unique common fixed point in $X$.

Proof. For any $x_{0} \in X$, we construct the sequence $\left(x_{n}\right)$ as $x_{n}=T_{n}\left(x_{n-1}\right), n=1,2, \cdots$. Using (1) and the homogeneity of $F$, we obtain

$$
\begin{aligned}
F\left(D\left(x_{1}, x_{2}, x_{2}\right)\right)= & F\left(D\left(T_{1}\left(x_{0}\right), T_{2}\left(x_{1}\right), T_{2}\left(x_{1}\right)\right)\right) \\
\leq & \left.\delta_{1,2}^{s} F\left(\left[D\left(x_{0}, T_{1}\left(x_{0}\right), T_{1}\left(x_{0}\right)\right)\right)+D\left(x_{1}, T_{2}\left(x_{1}\right), T_{2}\left(x_{1}\right)\right)\right]\right) \\
& +\gamma_{1,2}^{s} F\left(D\left(x_{0}, x_{1}, x_{1}\right)\right) \\
= & \delta_{1,2}^{s} F\left(\left[D\left(x_{0}, x_{1}, x_{1}\right)+D\left(x_{1}, x_{2}, x_{2}\right)\right]\right)+\gamma_{1,2}^{s} F\left(D\left(x_{0}, x_{1}, x_{1}\right)\right) .
\end{aligned}
$$

By the sub-additivity of $F$, we have

$$
\left(1-\delta_{1,2}^{s}\right) F\left(D\left(x_{1}, x_{2}, x_{2}\right)\right) \leq\left(\delta_{1,2}^{s}+\gamma_{1,2}^{s}\right) F\left(D\left(x_{0}, x_{1}, x_{1}\right)\right),
$$

i.e.

$$
F\left(D\left(x_{1}, x_{2}, x_{2}\right)\right) \leq\left(\frac{\delta_{1,2}^{s}+\gamma_{1,2}^{s}}{1-\delta_{1,2}^{s}}\right) F\left(D\left(x_{0}, x_{1}, x_{1}\right)\right) .
$$

Also, we get

$$
\begin{aligned}
F\left(D\left(x_{2}, x_{3}, x_{3}\right)\right) & =F\left(D\left(T_{2}\left(x_{1}\right), T_{3}\left(x_{2}\right), T_{3}\left(x_{2}\right)\right)\right) \\
& \leq\left(\frac{\delta_{2,3}^{s}+\gamma_{2,3}^{s}}{1-\delta_{2,3}^{s}}\right) F\left(D\left(x_{1}, x_{2}, x_{2}\right)\right) \\
& \leq\left(\frac{\delta_{2,3}^{s}+\gamma_{2,3}^{s}}{1-\delta_{2,3}^{s}}\right)\left(\frac{\delta_{1,2}^{s}+\gamma_{1,2}^{s}}{1-\delta_{1,2}^{s}}\right) F\left(D\left(x_{0}, x_{1}, x_{1}\right)\right) .
\end{aligned}
$$

By repeating the above process, we have

$$
F\left(D\left(x_{n}, x_{n+1}, x_{n+1}\right)\right) \leq \prod_{i=1}^{n}\left(\frac{\delta_{i, i+1}^{s}+\gamma_{i, i+1}^{s}}{1-\delta_{i, i+1}^{s}}\right) F\left(D\left(x_{0}, x_{1}, x_{1}\right)\right)=: C_{n} F\left(D\left(x_{0}, x_{1}, x_{1}\right)\right) .
$$

Hence we derive, by making use of the Lemma 2.1 and the properties of $F$, that for , $p \in \mathbb{N}, p>0$

$$
D\left(x_{n}, x_{n+p}, x_{n+p}\right) \leq K^{p-1}\left[D\left(x_{n}, x_{n+1}, x_{n+1}\right)+\cdots+D\left(x_{n+p-1}, x_{n+p}, x_{n+p}\right)\right]
$$

and so

$$
\begin{aligned}
F\left(D\left(x_{n}, x_{n+p}, x_{n+p}\right)\right) \leq & K^{(p-1) s}\left[F\left(D\left(x_{n}, x_{n+1}, x_{n+1}\right)\right)+F\left(D\left(x_{n+1}, x_{n+2}, x_{n+2}\right)\right)\right. \\
& \left.+\cdots+F\left(D\left(x_{n+p-1}, x_{n+p}, x_{n+p}\right)\right)\right] \\
\leq & K^{(p-1) s}\left[C_{n} F\left(D\left(x_{0}, x_{1}, x_{1}\right)\right)+C_{n+1} F\left(D\left(x_{0}, x_{1}, x_{1}\right)\right)\right. \\
& \left.+\cdots+C_{n+p-1} F\left(D\left(x_{0}, x_{1}, x_{1}\right)\right)\right] \\
= & K^{(p-1) s}\left[\sum_{k=0}^{p-1} C_{n+k} F\left(D\left(x_{0}, x_{1}, x_{1}\right)\right)\right] \\
= & K^{(p-1) s}\left[\sum_{k=n}^{n+p-1} C_{k}\right] F\left(D\left(x_{0}, x_{1}, x_{1}\right)\right) .
\end{aligned}
$$


Letting $n \rightarrow \infty$ and since $F^{-1}(0)=\{0\}$ and $F$ is continuous, we deduce that $D\left(x_{n}, x_{n+p}, x_{n+p}\right) \rightarrow 0$. Thus $\left(x_{n}\right)$ is a $G$-Cauchy sequence and, by $G$-completeness of $X$, converges to say $x^{*} \in X$. Moreover, for any natural number $m \neq 0$, we have

$$
\begin{aligned}
F\left(D\left(x_{n}, T_{m}\left(x^{*}\right), T_{m}\left(x^{*}\right)\right)\right)= & F\left(D\left(T_{n}\left(x_{n-1}\right), T_{m}\left(x^{*}\right), T_{m}\left(x^{*}\right)\right)\right) \\
\leq & \delta_{n, m}^{s}\left[F\left(D\left(x_{n-1}, x_{n}, x_{n}\right)\right)+F\left(D\left(x^{*}, T_{m}\left(x^{*}\right), T_{m}\left(x^{*}\right)\right)\right)\right] \\
& +\gamma_{n, m}^{s} F\left(D\left(x_{n-1}, x^{*}, x^{*}\right)\right) .
\end{aligned}
$$

Taking $\lim \sup$ as $n \rightarrow \infty$, we get

$$
F\left(D\left(x^{*}, T_{m}\left(x^{*}\right), T_{m}\left(x^{*}\right)\right)\right) \leq \delta_{n, m}^{s} F\left(D\left(x^{*}, T_{m}\left(x^{*}\right), T_{m}\left(x^{*}\right)\right)\right),
$$

and since $\limsup _{i \rightarrow \infty} \delta_{i, j}^{s}<1$, it follows that $F\left(D\left(x^{*}, T_{m}\left(x^{*}\right), T_{m}\left(x^{*}\right)\right)\right)=0$, i.e. $T_{m}\left(x^{*}\right)=x^{*}$. Then $x^{*}$ is a common fixed point of $\left\{T_{m}\right\}_{m>1}$.

To prove the uniqueness of $x^{*}$, let us suppose that $y^{*}$ is a common fixed point of $\left\{T_{m}\right\}_{m \geq 1}$, that is $T_{m}\left(y^{*}\right)=y^{*}$ for any $m \geq 1$. Then, by (1), we have

$$
\begin{aligned}
F\left(D\left(x^{*}, y^{*}, y^{*}\right)\right) & \leq F\left(D\left(T_{n}\left(x^{*}\right), T_{m}\left(y^{*}\right), T_{m}\left(y^{*}\right)\right)\right) \\
& \leq \delta_{n, m}^{s}\left[F \left(D\left(x^{*}, T_{n}\left(x^{*}\right), T_{n}\left(x^{*}\right)\right)+F\left(D\left(y^{*}, T_{m}\left(y^{*}\right), T_{m}\left(y^{*}\right)\right)\right]+\gamma_{n, m}^{s} F\left(D\left(x^{*}, y^{*}, y^{*}\right)\right)\right.\right. \\
& =\delta_{n, m}^{s}\left[F \left(D\left(x^{*}, x^{*}, x^{*}\right)+F\left(D\left(y^{*}, y^{*}, y^{*}\right)\right]+\gamma_{n, m}^{s} F\left(D\left(x^{*}, y^{*}, y^{*}\right)\right)\right.\right. \\
& =\gamma_{n, m}^{s} F\left(D\left(x^{*}, y^{*}, y^{*}\right)\right) .
\end{aligned}
$$

And again, since $0 \leq \gamma_{n, m}<1, x^{*}=y^{*}$. So $x^{*}$ is the unique common fixed point of $\left\{T_{m}\right\}$.

As particular cases of Theorem 2.2, we have the following two corollaries.

Corollary 2.3. Let $(X, D, K)$ be a G-complete $G$-metric type space and $\left\{T_{n}\right\}$ be a sequence of self mappings on $X$ such that

$$
\begin{aligned}
D\left(T_{i}(x), T_{j}(y), T_{j}(z)\right) \leq & \delta_{i, j}\left[D\left(x, T_{i}(x), T_{i}(x)\right)+D\left(y, T_{j}(y), T_{j}(z)\right)\right] \\
& +\gamma_{i, j} D(x, y, z)
\end{aligned}
$$

for $x, y, z \in X$ with $x \neq y, 0 \leq \delta_{i, j}, \gamma_{i, j}<1, i, j=1,2, \cdots$. If

i) for each $j, \limsup _{i \rightarrow \infty} \delta_{i, j}<1$,

ii)

$$
\sum_{n=1}^{\infty} C_{n}<\infty \text { where } C_{n}=\prod_{i=1}^{n} \frac{\delta_{i, i+1}+\gamma_{i, i+1}}{1-\delta_{i, i+1}}
$$

then $\left\{T_{n}\right\}$ has a unique common fixed point in $X$.

Proof. Apply Theorem 2.2 by putting $F=I_{[0, \infty)}$, the identity map.

Corollary 2.4. Let $(X, D, K)$ be a G-complete $G$-metric type space and $\left\{T_{n}\right\}$ be a sequence of self mappings on $X$ such that

$$
F\left(D\left(T_{i}(x), T_{j}(y), T_{j}(z)\right)\right) \leq F\left(\delta_{i, j}\left[D\left(x, T_{i}(x), T_{i}(x)\right)+D\left(y, T_{j}(y), T_{j}(z)\right)\right]\right)
$$

for $x, y, z \in X$ with $x \neq y, 0 \leq \delta_{i, j}<1, i, j=1,2, \cdots$, and for some $F \in \Phi$ homogeneous with degree s. If 
i) for each $j, \limsup _{i \rightarrow \infty} \delta_{i, j}^{s}<1$

ii)

$$
\sum_{n=1}^{\infty} C_{n}<\infty \text { where } C_{n}=\prod_{i=1}^{n} \frac{\delta_{i, i+1}^{s}}{1-\delta_{i, i+1}^{s}}
$$

then $\left\{T_{n}\right\}$ has a unique common fixed point in $X$.

Proof. Apply Theorem 2.2 by putting $\gamma_{i, i+1}=0$.

In the theory of fixed point, it is in general very difficult to find a non-trivial example to illustrate the main result. In the following lines, the trivial common fixed point is 0 but we prove that the condition (1) can really occur.

Example 2.5. (Compare [5])

Let $X=[0,1]$ and $D(x, y, z)=\max \{|x-y|,|y-z|,|z-x|\}$ whenever $x, y \in[0,1]$. Clearly, $(X, G)$ is a G-complete G-metric space. Following the notation in Theorem 2.2, we set $\delta_{i, j}=\left(\frac{1}{1+2^{i}}\right)^{2}$. We also define $T_{i}(x)=\frac{x}{4^{i}}$ for all $x \in X$ and $i=1,2, \cdots$ and $F:[0, \infty) \rightarrow[0, \infty), x \mapsto \sqrt{x}$. Then $F$ is continuous, non-decreasing, sub-additive and homogeneous of degree $s=\frac{1}{2}$ and $F^{-1}(0)=\{0\}$. Assume $i<j$ and $x>y \geq z$. Hence we have

$$
F\left(D\left(T_{i}(x), T_{j}(y), T_{j}(z)\right)\right)=\sqrt{\left|\frac{x}{4^{i}}-\frac{z}{4^{j}}\right|}
$$

and

$$
F\left(\delta_{i, j}\left[D\left(x, T_{i}(x), T_{i}(x)\right)+D\left(y, T_{j}(y), T_{j}(z)\right]\right)=\sqrt{\left(\frac{1}{1+2^{i}}\right)^{2}\left(\left|x-\frac{x}{4^{i}}\right|+\left|y-\frac{z}{4^{j}}\right|\right)} .\right.
$$

Therefore condition (4) is satisfied for all $x, y \in X$ with $x \neq y$. Moreover, since $F$ is homogeneous of degree $s=\frac{1}{2}$, we have

i) $\limsup _{i \rightarrow \infty} \delta_{i, j}<1$,

ii)

$$
C_{n}=\prod_{i=1}^{n} \frac{\delta_{i, i+1}^{s}}{1-\delta_{i, i+1}^{s}}=\prod_{i=1}^{n} \frac{1}{2^{i}}=\frac{1}{2^{\frac{n(n+1)}{2}}} \leq \frac{1}{2^{n}}
$$

The conditions of Corollary 2.4 are satisfied, $\left\{T_{n}\right\}$ has a common fixed point, which in this case is $x^{*}=0$.

Using the same idea as in the proof of Theorem 2.2, one can establish the following result.

Theorem 2.6. Let $(X, D, K)$ be a $G$-complete $G$-metric type space and $\left\{T_{n}\right\}$ be a sequence of self mappings on $X$ such that

$$
F\left(D\left(T_{i}(x), T_{j}(y), T_{j}(z)\right)\right) \leq F\left(\delta_{i, j}\left[D\left(x, T_{j}(y), T_{j}(z)\right)+D\left(y, T_{i}(x), T_{i}(x)\right)\right]\right)
$$

for $x, y, z \in X$ with $x \neq y, 0 \leq \delta_{i, j}, i, j=1,2, \cdots$, and for some $F \in \Phi$ homogeneous with degree s. If

i) for each $j, \limsup _{i \rightarrow \infty} \delta_{i, j}^{s}<1$,

ii)

$$
\sum_{n=1}^{\infty} C_{n}<\infty \text { where } C_{n}=\prod_{i=1}^{n} \frac{\delta_{i, i+1}^{s}+\gamma_{i, i+1}^{s}}{1-\delta_{i, i+1}^{s}}
$$

then $\left\{T_{n}\right\}$ has a unique common fixed point in $X$. 
Corollary 2.7. In addition to the hypotheses of Theorem 2.6, supposed that for every $n \in \mathbb{N}$, there exists a $k_{n} \in \mathbb{N}$ such that $\delta_{n, k_{n}}^{s}<\frac{1}{1+2^{s}}$, then every $T_{n}$ has a unique fixed point in $X$.

Proof. We shall make use of [iii)] of Definition 1.6. According to Theorem $2.6\left\{T_{n}\right\}$ has a unique common fixed point $u \in X$. If $v$ is a fixed point for $T_{m}$, then

$$
\begin{aligned}
F(D(u, v, v))=F\left(D\left(T_{k_{m}} u, T_{m} v, T_{m} v\right)\right) & \leq F\left(\delta_{m, k_{m}}\left[D\left(u, T_{m} v, T_{m} v\right)+D\left(v, T_{k_{m}} u, T_{k_{m}} u\right)\right]\right) \\
& \leq F\left(\delta_{m, k_{m}}[D(u, v, v)+D(v, u, u)]\right) \\
& \leq \delta_{m, k_{m}}^{s}\left[F(D(u, v, v))+2^{s} F(D(u, v, v))\right] \\
& \leq \delta_{m, k_{m}}^{s}\left[1+2^{s}\right] F(D(u, v, v))
\end{aligned}
$$

which implies that $D(u, v, v)=0$. Hence $u=v$.

\section{More Results}

We shall conclude this article with the following interesting result.

Theorem 3.1. Let $(X, D, K)$ be a $G$-complete $G$-metric type space and $\left\{T_{n}\right\}$ be a sequence of self mappings on $X$ such that

$$
\begin{aligned}
F\left(D\left(T_{i}(x), T_{j}(y), T_{j}(z)\right)\right) \leq & F\left(\delta_{i, j} D\left(y, T_{j} y, T_{j} z\right) \phi\left(D\left(x, T_{i} x, T_{i} x\right), D(x, y, z)\right)\right) \\
& +F\left(\gamma_{i, j} D(x, y, z)\right)
\end{aligned}
$$

for $x, y, z \in X$ with $x \neq y, 0 \leq \delta_{i, j}, \gamma_{i, j} ; 0 \leq \delta_{i, j}+\gamma_{i, j}<1, i, j=1,2, \cdots, i \neq j$ where $\phi:[0, \infty) \times[0, \infty) \rightarrow$ $[0, \infty)$ is a continuous function such that $\phi(t, t)=1$ for all $t \in[0, \infty)$ and for some $F \in \Phi$ homogeneous with degree $s$. If

i) for each $j, \limsup _{i \rightarrow \infty} \delta_{i, j}^{s}<1$, and $\limsup _{i \rightarrow \infty} \gamma_{i, j}^{s}<1$,

ii)

$$
\sum_{n=1}^{\infty} C_{n}<\infty \text { where } C_{n}=\prod_{i=1}^{n} \frac{\gamma_{i, i+1}^{s}}{1-\delta_{i, i+1}^{s}}
$$

then $\left\{T_{n}\right\}$ has a unique common fixed point in $X$.

Proof. For any $x_{0} \in X$, we construct the sequence $\left(x_{n}\right)$ as $x_{n}=T_{n}\left(x_{n-1}\right), n=1,2, \cdots$. Using (6) and the properties of $F$, we obtain

$$
\begin{aligned}
F\left(D\left(x_{1}, x_{2}, x_{2}\right)\right)= & F\left(D\left(T_{1}\left(x_{0}\right), T_{2}\left(x_{1}\right), T_{2}\left(x_{1}\right)\right)\right) \\
\leq & \delta_{1,2}^{s} F\left(D\left(x_{1}, x_{2}, x_{2}\right) \phi\left(D\left(x_{0}, x_{1}, x_{1}\right), D\left(x_{0}, x_{1}, x_{1}\right)\right)\right) \\
& +\gamma_{1,2}^{s} F\left(D\left(x_{0}, x_{1}, x_{1}\right)\right) \\
\leq & \delta_{1,2}^{s} F\left(D\left(x_{1}, x_{2}, x_{2}\right)\right)+\gamma_{1,2}^{s} F\left(D\left(x_{0}, x_{1}, x_{1}\right)\right)
\end{aligned}
$$

which implies

$$
F\left(D\left(x_{1}, x_{2}, x_{2}\right)\right) \leq \frac{\gamma_{1,2}^{s}}{1-\delta_{1,2}^{s}} F\left(D\left(x_{0}, x_{1}, x_{1}\right)\right) .
$$

Moreover, it is easy to see that

$$
F\left(D\left(x_{2}, x_{3}, x_{3}\right)\right) \leq \frac{\gamma_{2,3}^{s}}{1-\delta_{2,3}^{s}} \times \frac{\gamma_{1,2}^{s}}{1-\delta_{1,2}^{s}} F\left(D\left(x_{0}, x_{1}, x_{1}\right)\right)
$$


Generally, we conclude that

$$
F\left(D\left(x_{n}, x_{n+1}, x_{n+1}\right)\right) \leq \prod_{i=1}^{n} \frac{\gamma_{i, i+1}^{s}}{1-\delta_{i, i+1}^{s}} F\left(D\left(x_{0}, x_{1}, x_{1}\right)\right)=: C_{n} F\left(D\left(x_{0}, x_{1}, x_{1}\right)\right) .
$$

Therefore, for $p \in \mathbb{N}, p>0$ we get

$$
\begin{aligned}
F\left(D\left(x_{n}, x_{n+p}, x_{n+p}\right)\right) \leq & K^{(p-1) s}\left[F\left(D\left(x_{n}, x_{n+1},, x_{n+1}\right)\right)+F\left(D\left(x_{n+1}, x_{n+2}, x_{n+2}\right)\right)\right. \\
& \left.+\ldots+F\left(D\left(x_{n+p-1}, x_{n+p}, x_{n+p}\right)\right)\right] \\
= & K^{(p-1) s}\left[\sum_{k=n}^{n+p-1} C_{k} F\left(D\left(x_{0}, x_{1}, x_{1}\right)\right)\right]
\end{aligned}
$$

Letting $n \rightarrow \infty$ and since $F^{-1}(0)=\{0\}$ and $F$ is continuous, we deduce that $\left(x_{n}\right)$ is a $G$-Cauchy sequence and, by $G$-completeness of $X$, converges to say $x^{*} \in X$. Now for any positive integer $m$,

$$
\begin{aligned}
F\left(D\left(x_{n}, T_{m} x^{*}, T_{m} x^{*}\right)\right) & =F\left(D\left(T_{n}\left(x_{n-1}, T_{m} x^{*}, T_{m} x^{*}\right)\right)\right. \\
& \leq \delta_{n, m}^{s} F\left(D\left(x^{*}, T_{m} x^{*}, T_{m} x^{*}\right) \phi\left(D\left(x_{n-1}, x_{n}, x_{n}\right), D\left(x_{n-1}, x^{*}, x^{*}\right)\right)\right) \\
& +\gamma_{n, m}^{s} F\left(D\left(x_{n-1}, x^{*}, x^{*}\right)\right) .
\end{aligned}
$$

Taking $\lim \sup$ as $n \rightarrow \infty$, it follows that $x^{*}=T_{m} x^{*}$, i.e. $x^{*}$ is a common fixed point for $\left\{T_{n}\right\}$. For uniqueness, assume $y^{*}$ is a common fixed point for $\left\{T_{n}\right\}$, then

$$
\begin{aligned}
F\left(D\left(x^{*}, y^{*}, y^{*}\right)\right) & =F\left(D\left(T_{n} x^{*}, T_{m} y^{*}, T_{m} y^{*}\right)\right) \\
& \leq \delta_{n, m}^{s} F\left(D\left(y^{*}, T_{m} y^{*}, T_{m} y^{*}\right) \phi\left(D\left(x^{*}, T_{n} x^{*}, T_{n} x^{*}\right), D\left(x^{*}, y^{*}, y^{*}\right)\right)\right) \\
& +\gamma_{n, m}^{s} F\left(D\left(x^{*}, y^{*}, y^{*}\right)\right) \\
& =\delta_{n, m}^{s} F\left(D\left(y^{*}, y^{*}, y^{*}\right) \phi\left(D\left(x^{*}, x^{*}, x^{*}\right), D\left(x^{*}, y^{*}, y^{*}\right)\right)\right) \\
& +\gamma_{n, m}^{s} F\left(D\left(x^{*}, y^{*}, y^{*}\right)\right) \\
& =\gamma_{n, m}^{s} F\left(D\left(x^{*}, y^{*}, y^{*}\right)\right) .
\end{aligned}
$$

Taking limsup as $n \rightarrow \infty$, we get $x^{*}=y^{*}$.

\section{References}

1. Z. Mustafa, H. Obiedat, and F. Awawdeh, "Some fixed point theorem for mapping on complete g-metric spaces," Fixed point theory and Applications, vol. 2008, no. 1, pp. 1-12, 2008.

2. Z. Mustafa and B. Sims, "A new approach to generalized metric spaces," Journal of Nonlinear and convex Analysis, vol. 7, no. 2, pp. 289-297, 2006.

3. B. C. Dhage, "Generalised metric space and mappings with fixed point," 1992.

4. Y. U. Gaba, " $\lambda$-sequences and fixed point theorem in G-metric type spaces," 2016, manuscript submitted for publication.

5. - "Metric type spaces and $\lambda$-sequences," Quaestiones Mathematicae, (in press).

6. O. S. Iyiola and Y. U. Gaba, "On metric type spaces and fixed point theorems," Applied Mathematical Sciences, vol. 8, no. 79, pp. 3905-3920, 2014.

7. M. Jleli and B. Samet, "Remarks on g-metric spaces and fixed point theorems," Fixed Point Theory and Applications, vol. 2012, no. 1, pp. 1-7, 2012.

8. S. K. Mohanta, "Some fixed point theorems in g-metric spaces," An. Stiint. Univ. Ovidius, Constanta. Ser. Mat, vol. 20, no. 1, pp. 285-306, 2012. 